\title{
Efeito da suplementação com selênio e com as vitaminas C e $E$ sobre biomarcadores hematológicos em militares durante treinamento físico vigoroso e prolongado
}

Effect of supplementation with selenium and vitamins $C$ and $E$ on hematological biomarkers in military personnel during intense and prolonged physical training

\author{
Márcio Sena \\ Instituto de Pesquisa da Capacitação Física do Exército \\ mabsmarcio@gmail.com \\ Marcos Fortes \\ Instituto de Pesquisa da Capacitação Física do Exército \\ msrfortes@gmail.com \\ Danielli Mello \\ Instituto de Pesquisa da Capacitação Física do Exército \\ danielli.mello@gmail.com \\ Sérgio Lisboa \\ Instituto de Pesquisa da Capacitação Física do Exército \\ machadosı@globo.com \\ Marcos Pereira \\ Universidade Federal do Rio de Janeiro \\ marcosdpufrj@gmail.com
}

Conflito de interesses: nada a declarar. Financiamento: nada a declarar. 


\section{RESUMO}

Neste estudo foi investigado o efeito da suplementação com selênio, vitaminas C e E sobre biomarcadores hematológicos em militares submetidos a treinamento físico intenso e prolongado. Participaram 37 homens (32,0 \pm 7,2 anos), durante 37 dias. Dois grupos foram separados aleatoriamente: Grupo Suplementado (GS) e Grupo Placebo (GP). 0 GS ingeriu cápsulas contendo selênio quelado $(0,06 \mathrm{mg})$, vitamina $\mathrm{C}(1000 \mathrm{mg})$ e vitamina $\mathrm{E}$ (500 mg) e, o GP ingeriu (1500 mg de amido). 0 sangue foi coletado antes do início do estudo (T0); ao final da primeira (T1) e terceira (T2) semanas de treinamento. Os dados foram analisados pela ANOVA 3X2 $(p<0,05)$. A hemoglobina reduziu em T1 e T2 no GS e GP versus T0. A hemoglobina corpuscular média reduziu em T1 e T2 no GS e no GP apenas em T2 versus T0. A concentração da hemoglobina corpuscular média reduziu no GS em T1 e T2 versus T0, porém no GP reduziu em T2 versus T0 e T1. As hemácias, hematócrito, volume corpuscular médio e a amplitude de distribuição dos glóbulos vermelhos não apresentaram diferenças significativas. 0 treinamento físico induziu uma diminuição na hemoglobina dos militares, e a suplementação não promoveu proteção contra este dano.

Palavras-Chave: antioxidantes, estresse oxidativo, exercício físico, hemograma, militares

\section{ABSTRACT}

In this study, we investigated the effect of supplementation with selenium, vitamins $C$ and $\mathrm{E}$ on hematological biomarkers in military personnel undergoing intense and prolonged physical training (IPPT). Thirty-seven men (32.0 \pm 7.2 years) participated for 37 days. Two groups were randomly separated: Supplemented Group (SG) and Placebo Group (PG). The SG $(n=13)$ ingested capsules containing chelated selenium $(0.06 \mathrm{mg})$, vitamin $C$ (1000 $\mathrm{mg}$ ) and vitamin $\mathrm{E}(500 \mathrm{mg})$ and the PG: ( $\mathrm{n}=11)$ ingested (1500 mg of starch). Blood samples were collected before the start of the study (T0); at the end of the first (T1) and third (T2) weeks of IPPT. Data was analyzed by ANOVA 3X2 $(p<0.05)$. Hemoglobin has decreased in T1 and T2 in SG and PG versus T0. Mean corpuscular hemoglobin reduced in $\mathrm{T} 1$ and $\mathrm{T} 2$ in $\mathrm{SG}$ and in $\mathrm{PG}$ only in T2 versus T0. The mean corpuscular hemoglobin concentration decreased in SG at T1 and T2 versus T0, but in PG it reduced at T2 versus T0 and T1. No significant differences have been observed with red blood cells, hematocrit, mean corpuscular volume and red cell distribution width. The physical training induced a decrease in hemoglobin and supplementation did not provide protection against this damage.

Keywords: antioxidants, oxidative stress, physical exercise, blood count, military 


\section{INTRODUÇÃO}

0 treinamento militar, particularmente aqueles realizados em cursos operacionais das forças armadas são realizados sob alta intensidade e grande volume exigindo uma grande demanda energética, que pode levar a depleção dos nutrientes que são essenciais ao bom funcionamento do organismo, dos sistemas antioxidante e imunológico, trazendo comprometimentos à saúde do militar (Müller-Schilling et al., 2019; Ojanen et al., 2018; Szivak et al., 2018).

Neste contexto, está bem estabelecido na literatura que o exercício físico intenso e prolongado (EFIP) induz o aumento no consumo de oxigênio $\left(\mathrm{O}_{2}\right)$, tendo como consequência uma maior produção das espécies reativas de oxigênio (EROs), favorecendo a instalação de um quadro de estresse oxidativo (EO) (Di Meo et al., 2019; Marengo et al., 2016). Por outro lado, a prática de exercício físico (EF) moderado está associada com inúmeros benefícios para saúde, contribuindo para o aumento da qualidade de vida e redução no risco do desenvolvimento de muitas doenças, tais como diabetes, hipertensão e doença cardiovascular (DCV) (Danese et al., 2017; Dasso, 2019).

0 EO ocorre quando existe um desequilíbrio entre os sistemas pró-oxidantes e antioxidantes, de forma que os primeiros predominem, levando ao comprometimento na sinalização celular, controle das reações de oxirredução (redox) e do reparo ao dano molecular (Pisoschi \& Pop, 2015; Sies, 2018). Neste cenário, as estruturas mais suscetíveis ao ataque das EROs são as proteínas, os lipídios e o ácido desoxirribonucleico (DNA), que sofrem alterações na estrutura, resultando em perda de funcionalidade (Di Meo et al., 2019; Marengo et al., 2016). As moléculas oxidantes mais comuns são o peróxido de hidrogênio $\left(\mathrm{H}_{2} \mathrm{O}_{2}\right)$, radicais superóxido $\left(\mathrm{O}_{2}{ }^{-}\right)$e hidroxila $\left(\mathrm{OH}^{\circ}\right)$, todas oriundas do metabolismo do oxigênio $\left(\mathrm{O}_{2}\right)$ (Marengo et al., 2016). Destacam-se como as três principais fontes endógenas produtoras de EROs durante o EF: a mitocôndria, o mecanismo de isquemia e reperfusão e a resposta inflamatória (Marengo et al., 2016; Pisoschi \& Pop, 2015).

Convém ressaltar, que as enzimas nicotinamida adenina dinucleotídeo fosfato (NADPH) oxidase constituem uma das principais fontes de produção de EROs durante a contração muscular, principalmente ânion superóxido (02*-) e peróxido de hidrogênio $\left(\mathrm{H}_{2} \mathrm{O}_{2}\right)$, tendo a sua expressão aumentada durante o treinamento físico (Brendel et al., 2020; Tarafdar \& Pula, 2018). Ao mesmo tempo, é sabido que a sua presença nas células fagocitárias contribui para aumentar o EO no organismo (Tarafdar \& Pula, 2018). Segundo Cunha et al. (2017), o treinamento físico aeróbio reduz a atividade das NADPH oxidases muscular estando associada à redução das EROs e inflamação sistêmica. 
A fim de atenuar os danos causados pelas EROs, o organismo possui dois grandes sistemas antioxidantes: sistema enzimático, que faz parte as enzimas superóxido dismutase (SOD), glutationa peroxidase (GPx) e catalase e; sistema não enzimático, composto por pequenas moléculas que podem ser adquiridas pela alimentação ou sintetizadas pelo próprio organismo, tais como glutationa (GSH), bilirrubina, ácido úrico $(\mathrm{AU})$, ácido $\alpha$-lipóico, $\beta$-caroteno (precursor de vitamina $\mathrm{A}$ ), ácido ascórbico (vitamina $\mathrm{C}$ ), a-tocoferol (vitamina $E$ ), selênio $(\mathrm{Se})$, cobre $(\mathrm{Cu})$, zinco $(\mathrm{Zn})$, magnésio e flavonóides (Marengo et al., 2016; Pisoschi \& Pop, 2015; Poblete-Aro et al., 2018; Viña et al., 2020). Os antioxidantes são descritos como substâncias que, mesmo em baixas concentrações tem a propriedade de retardar ou inibir a oxidação do substrato oxidável (Cotinguiba et al., 2013). Sua classificação é de acordo com o seu mecanismo de ação, dividindo-se em prevenção, interceptação e reparo (Helmut Sies, Carsten Berndt, 2017).

Segundo Valko et al. (2016), a membrana plasmática parece ser mais suscetível ao ataque de EROs, em decorrência da oxidação dos lipídeos, processo conhecido como peroxidação lipídica (PL) ou lipoperoxidação. Neste cenário, sabe-se que a hemácia é uma das principais células vulneráveis a este ataque, devido ao papel fisiológico desempenhado bem como a presença em grande quantidade no organismo (Lücker et al., 2017). A ocorrência destes danos é exacerbada pelo EFIP, que induz os estresses mecânicos (lise intravascular ou extravascular) e metabólico (aumento das EROs), ambos favorecendo a destruição da membrana das hemácias (hemólise) e o desenvolvimento da PL (Gaschler \& Stockwell, 2018; Rifkind et al., 2015).

A hemólise desencadeia uma série de complicações para o organismo, dentre as quais destacam-se: a diminuição no aporte de oxigênio $\left(\mathrm{O}_{2}\right)$ para os tecidos (hipóxia), liberação de hemoglobina $(\mathrm{Hb})$ e ferro $\left(\mathrm{Fe}^{2+}\right)$ do seu interior, podendo este íon ser catalisador da reação de Fenton, gerando mais EROs contribuindo para que mais hemácias possam ser destruídas (Rapido, 2017; Rifkind et al., 2015). Cabe ressaltar que danos provocados na membrana das hemácias jovens reduzem o tempo da célula na circulação sanguínea, pois serão consideradas senescentes e fagocitadas por macrófagos no baço, fígado e na medula óssea (Kumar \& Rizvi, 2014; Mairbäurl, 2013; Rapido, 2017). Convém salientar, que em hipóxia, a Hb é parcialmente oxigenada, o que contribui para sua auto-oxidação, deixando as células deficientes de $\mathrm{O}_{2}$ (Rifkind et al., 2015).

Alguns estudos reportam que dietas enriquecidas com moléculas antioxidantes trazem benefícios para o sistema antioxidante, contribuindo para o seu bom funcionamento (Balushi et al., 2019; Barbosa et al., 2010; Gammone et al., 2014). Neste contexto, destaca-se o selênio, as vitaminas $\mathrm{C}$ e $\mathrm{E}$, que também possuem atividade antioxidante $\mathrm{e}$ protegem as células do organismo contra danos oxidativos causados pela ação das EROs (Aguiló et al., 2007; Chou et al., 2018; Hasani et al., 2019). Considerando que o treinamento militar demanda grande gasto energético e leva ao aumento da produção de EROS, faz-se necessária o uso de suplemento que diminua a ação das mesmas. Diante 
desse cenário, o presente estudo objetivou investigar o efeito da suplementação de selênio e vitaminas $\mathrm{C}$ e E contra a ação das moléculas oxidativas em militares.

\section{MÉTODOS}

\section{Desenho de estudo, amostra e aspectos éticos}

A presente pesquisa trata-se de um estudo experimental, randomizado, duplo-cego e controlado por placebo com 37 militares do sexo masculino (32 $\pm 7,2$ anos), voluntários do curso básico paraquedista (CBP) oriundos de diversas organizações militares do Exército Brasileiro. Os critérios de inclusão no estudo foram o bom estado de saúde diagnosticado por uma avaliação médica prévia, assinatura do termo de consentimento livre e esclarecido (TCLE) e restrição de suplementação antioxidante, pois poderiam afetar a determinação dos parâmetros estudados, enquanto os critérios de exclusão foram a ausência em alguma etapa e/ou algum quadro clínico que impedisse a realização das avaliações.

Os militares foram divididos aleatoriamente em dois grupos: (i) suplementado (GS) $(n=$ 19), que recebeu administração de cápsulas contendo selênio quelado (0,06 mg), vitamina C $(1,000 \mathrm{mg})$ e vitamina $E(500 \mathrm{mg})$, e (ii) placebo (GP) $(n=18)$, que recebeu administração de $1,500 \mathrm{mg}$ de amido. A dose dos respectivos suplementos que foram utilizados no estudo seguiu as normas de recomendações diárias de consumo segundo Padovani et al. (2006).

A pesquisa atendeu às Normas para a Realização de Pesquisa em Seres Humanos, Resolução 466/12, do Conselho Nacional de Saúde de 12/12/2012, sendo submetido a Comissão Nacional de Ética em Pesquisa por meio da Plataforma Brasil e aprovado sob o número 028.3.2007/2008.

\section{Procedimentos de coleta de dados}

A coleta de dados ocorreu na área de estágio paraquedista do Exército Brasileiro, Rio de Janeiro. Os participantes de ambos os grupos fizeram uso do suplemento ou placebo por 37 dias, sendo este período dividido em 18 dias sem exercício físico intenso e 19 dias com exercício físico intenso. As coletas foram realizadas um dia antes do início do uso da suplementação, coleta basal (T0), ao final da primeira (T1) e terceira (T2) semanas de treinamento com exercício físico intenso (Figura 1). 
Figura 1. Desenho experimental referente aos períodos de treinamento e coletas de sangue durante 0 estudo

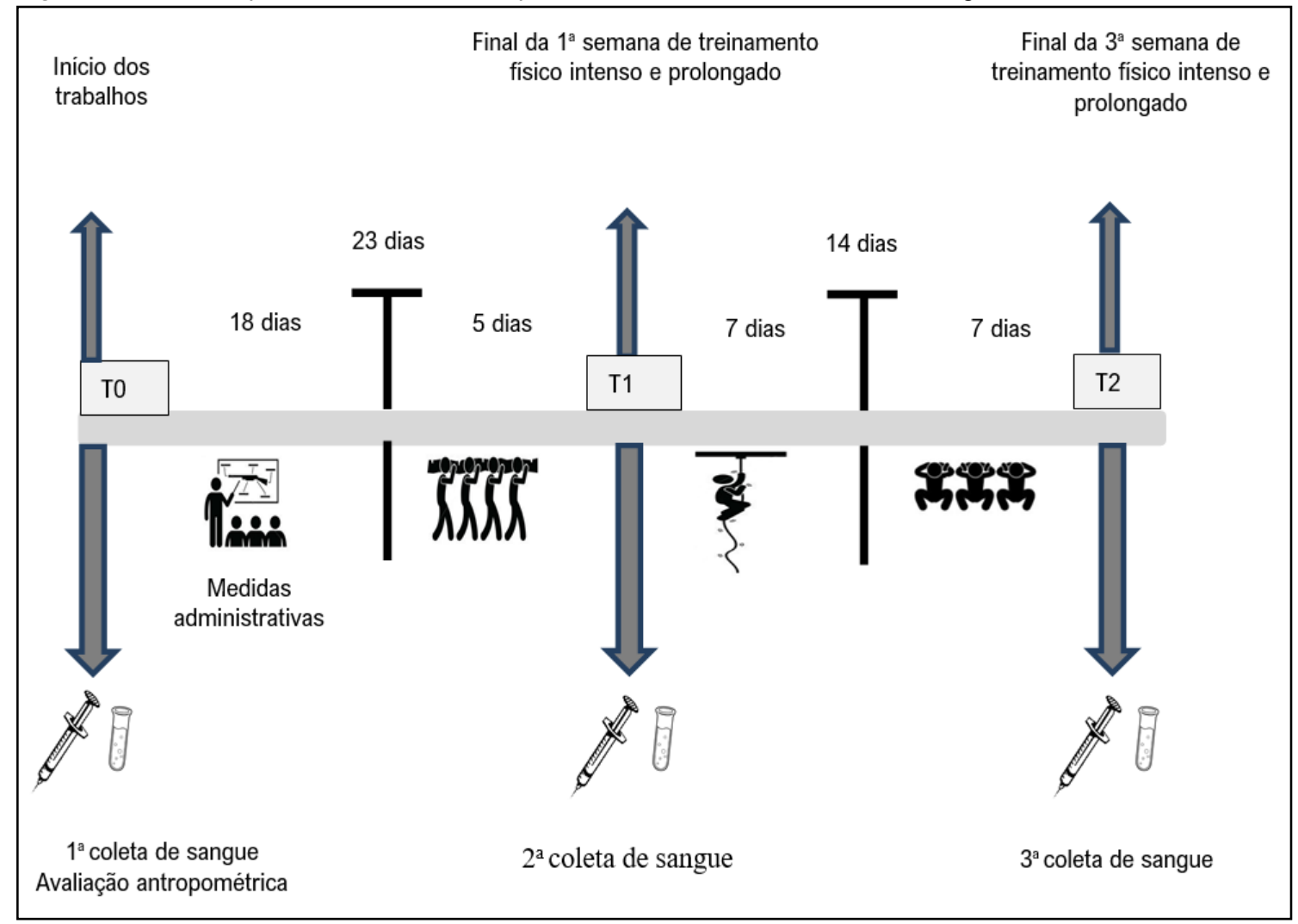

Fonte: Elaboração própria

Para obtenção do material biológico foi necessária à coleta de três tubos de $4 \mathrm{~mL}$, um em cada período, contendo o anticoagulante ácido etilenodiamino tetra-acético (EDTA) que foram usados para as análises dos parâmetros hematológicos, hemácias, Hb, Hct, volume corpuscular médio (VCM), hemoglobina corpuscular média (HCM), concentração de hemoglobina corpuscular média (CHCM) e RDW.

Os biomarcadores citados se encontram inseridos no exame clínico hemograma completo e foram dosados em sangue total no analisador hematológico ABX Micros $60^{\circledR}$ (HORIBA, Medical, Quioto, Japão), seguindo o protocolo estabelecido pelo fabricante do aparelho. As análises foram realizadas no laboratório de Análises Clínicas do Instituto de Pesquisa da Capacitação Física do Exército (IPCFEx) por um farmacêutico bioquímico. Os procedimentos das fases pré-analítica, analítica e pós-analíticas seguiram as recomendações da Sociedade Brasileira de Patologia Clínica e Medicina Laboratorial (Sumita et al., 2020).

Para a determinação da composição corporal foram utilizadas as variáveis massa corporal total, estatura, índice de massa corporal (IMC) e dobras cutâneas (DC). Para tal, foi utilizado o protocolo da Sociedade internacional para o avanço da Cineantropometria International Society for the Advancement of Kinanthropometry (ISAK) (Marfell-Jones et 
al., 2019). Para massa corporal total e estatura foi utilizada uma balança digital da marca Filizola ${ }^{\circledR}$ calibrada, com precisão de $0,1 \mathrm{Kg}$ e capacidade de $200 \mathrm{Kg}$, (MS, Brasil), e um estadiômetro da marca Sanny ${ }^{\circledR}$ com precisão de 0,001 m e capacidade de $210 \mathrm{~cm}$, (SP, Brasil). Posteriormente, foi calculado o IMC dividindo a massa corporal total pela estatura elevada ao quadrado $\left(\mathrm{Kg} / \mathrm{m}^{2}\right)(\mathrm{WHO}, 2020)$ A mensuração das DC peito, abdome e coxa, mensuradas com adipômetro Lange ${ }^{\circledR}$ com precisão de $1 \mathrm{~mm}$ (Cambridge, Maryland) e para o cálculo do percentual de gordura foi utilizado o protocolo de 3DC de Pollock e Wilmore (1993).

\section{Protocolo de exercício físico intenso e prolongado}

0 protocolo de EFIP foi realizado durante três semanas, de $2^{\mathrm{a}}$ a $6^{\mathrm{a}}$ feira (carga horária semanal de $15 \mathrm{~h}$ ), no período da manhã ( $7 \mathrm{~h} 15 \mathrm{~min}$ às $11 \mathrm{~h} 30 \mathrm{~min})$, sendo o período da tarde destinado à recuperação. As atividades realizadas correspondem a três seções de prática de EFIP, com a duração de uma hora cada, segundo Ainsworth et al. (2011), e intensidade muito intensa a máxima segundo classificação da escala de esforço percebido de Borg (Borg, 2000), com dispêndio energético equivalente a doze METs (equivalente metabólico).

Os exercícios realizados fazem parte da grade curricular do CBP e contemplam as seguintes atividades: Corrida contínua de $5 \mathrm{Km}$ em forma (juntos), pista de treinamento em circuito (PTC) (sete exercícios), ginástica básica (onze exercícios), ginástica com touros (seis exercícios) e pista de cordas (sete obstáculos). Os exercícios visam desenvolver as valências físicas inerentes as atividades desenvolvidas pelo paraquedista (Brasil, 2013). Durante os treinamentos foram utilizados os uniformes de características militar: Calça camuflada, coturno e sem camisa. Quando os exercícios foram realizados na pista de cordas, o aluno utilizou a gandola (casaco de combate).

\section{Análise estatística}

Os resultados da estatística descritiva das variáveis contínuas estão apresentados como média e desvio padrão. Os dados foram testados quanto à normalidade por meio do teste de Shapiro-Wilk e, apresentaram distribuição paramétrica, sendo a comparação intragrupos testada pela ANOVA com medidas repetidas no segundo fator, seguida do pós-teste de Bonferroni para verificar onde estão as diferenças encontradas. Para a comparação intergrupos foi utilizada a ANOVA Fatorial, considerando os fatores tempo e uso de suplementação ou placebo e nível de significância $p<0,05$. A análise estatística foi processada com auxílio do software STATISTICA ${ }^{\circledR}$ 12.0. 


\section{RESULTADOS}

\section{Amostragem e análise da composição corporal}

0 presente estudo não observou em nenhum biomarcador diferença estatística intergrupos. 0 início contou com a presença de trinta e sete voluntários (100\%), dos quais seis $(16,22 \%)$ foram desligados na primeira semana do curso (T1), sendo três do GS $(8,11 \%)$ e, três do GP $(8,11 \%)$. Após a primeira semana do curso (T2), dois voluntários foram desligados $(5,4 \%)$, sendo um do GS $(2,7 \%)$ e um do GP $(2,7 \%)$, perfazendo um total de oito desligamentos $(21,62 \%)$. Cabe ressaltar, que cinco voluntários não constam nesta análise devido a problemas na coleta do material biológico (13,5\%). De acordo com a Tabela 1, podemos observar as principais características de composição corporal dos voluntários do CBP. Os valores médios encontrados estão abaixo do ponto de corte considerado normal estabelecido na literatura, sendo 15\% para o percentual de gordura corporal (\% GC) e $25 \mathrm{~kg} / \mathrm{m}^{2}$ para o índice de massa corporal (IMC) (Marfell-Jones et al., 2019; Pollock \& Wilmore, 1993).

Tabela 1. Características antropométricas dos voluntários participantes de cada grupo em estudo

\begin{tabular}{|c|c|c|}
\hline \multirow[t]{2}{*}{ Características } & \multicolumn{2}{|l|}{ Grupo $(\mu \pm D P)$} \\
\hline & Suplementado $(n=13)$ & Placebo $(n=11)$ \\
\hline Idade (anos) & $30,9 \pm 8,1$ & $33,1 \pm 6,3$ \\
\hline Massa corporal (Kg) & $75,1 \pm 8,9$ & $74,6 \pm 8,2$ \\
\hline Estatura $(m)$ & $1,78 \pm 5,3$ & $1,76 \pm 8,1$ \\
\hline Índice de massa corporal (IMC) & $23,8 \pm 2,1$ & $24,2 \pm 2,5$ \\
\hline Percentual de gordura (\%) & $16,5 \pm 6,3$ & $14,3 \pm 3,8$ \\
\hline
\end{tabular}

média: $\boldsymbol{\mu}$; DP: desvio padrão.

Na Figura 2 é possível observar alterações induzidas pelo EFIP sobre a concentração de $\mathrm{Hb}$. Ocorreu redução no GS no T1 $(\Delta \%=-8,9 ; p=0,015)$ e T2 $(\Delta \%=-12,1 ; p=0,001)$ quando comparado com os valores obtidos antes do início do curso (T0) (Figura. 2A). 0 GP demonstrou um comportamento semelhante, tendo uma redução de $\mathrm{Hb}$ no período $\mathrm{T} 1(\Delta \%=-8,9 ; p=0,028)$ e T2 $(\Delta \%=-13,2 ; p=0,001)$, quando comparado com os valores obtidos antes do início do curso (T0) (Figura. 2B). 
Figura 2. Efeito do exercício físico vigoroso e prolongado, sobre a concentração de hemoglobina no sangue periférico. Os valores estão expressos como média \pm desvio padrão. Cada círculo fechado significa o valor obtido de hemoglobina de cada indivíduo do estudo. ( $\left.{ }^{a}\right)$ Quando estatisticamente significativo versus T0. $P<0,05$.

A)

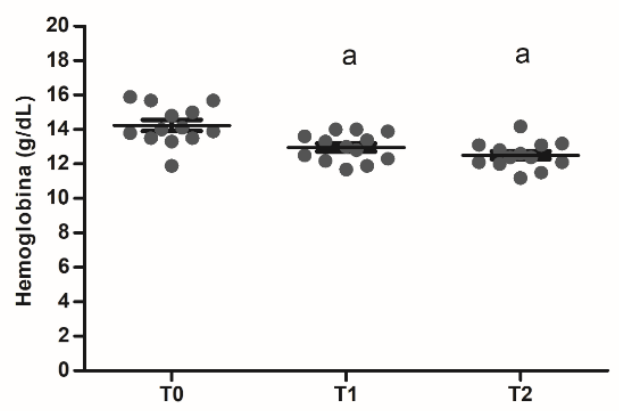

B)

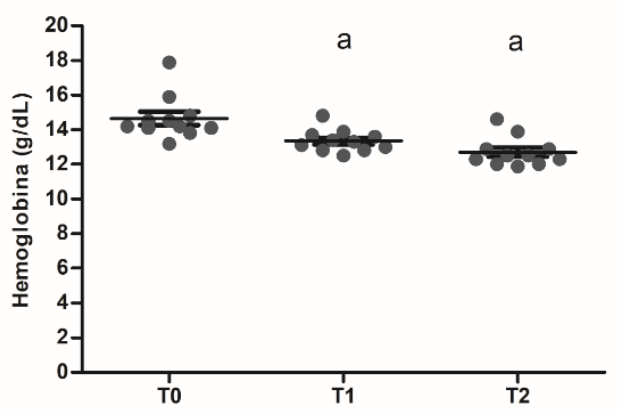

A Tabela 2 apresenta o efeito do EFIP sobre a concentração dos parâmetros hematológicos, onde observa-se redução na concentração da HCM no período $\mathrm{T} 1(\Delta \%=$ $-4,9 ; p=0,018)$ e T2 $(\Delta \%=-7,0 ; p=0,001)$ do GS, bem como no período T2 do GP $(\Delta \%$ $=-6,9 ; p=0,001)$ quando comparado com os valores basais obtidos antes do início do curso (T0). A CHCM demonstrou um comportamento semelhante ao observado na concentração de HCM do GS, reduzindo no período T1 $(\Delta \%=-2,7 ; p=0,001)$ e T2 $(\Delta \%=$ $-7,2 ; p=0,001)$ quando comparado com os valores basais obtidos antes do início do curso (T0). Por outro lado, o GP demostrou um comportamento diferente do observado na análise da concentração de $\mathrm{HCM}$, sendo observado na $\mathrm{CHCM}$ uma redução no período T2 $(\Delta \%=-7,2 ; p=0,001)$ quando comparado aos valores obtidos antes do início do curso (T0) e, outra redução neste mesmo período quando comparado com os valores obtidos na primeira semana de treinamento (T1) $(\Delta \%=-4,6 ; p=0,004)$. Em relação à concentração de hemácias, Hct, VCM e RDW não observamos diferença estatística ao longo dos períodos de treinamento deste estudo (Tabela 2).

Tabela 2. Valores máximos dos parâmetros hematológicos em diferentes períodos do treinamento físico.

\begin{tabular}{|c|c|c|c|c|}
\hline Biomarcadores & Grupo & TO & T1 & T2 \\
\hline \multirow{2}{*}{ Hemácias $\left(10^{6} / \mathrm{mm}^{3}\right)$} & suplementado & $4,75 \pm 0,41$ & $4,47 \pm 0,31$ & $4,43 \pm 0,32$ \\
\hline & placebo & $4,92 \pm 0,50$ & $4,63 \pm 0,45$ & $4,60 \pm 0,47$ \\
\hline \multirow[b]{2}{*}{ Hct (\%) } & suplementado & $40,90 \pm 2,90$ & $39,24 \pm 2,37$ & $38,91 \pm 2,49$ \\
\hline & placebo & $43,26 \pm 3,58$ & $40,50 \pm 2,63$ & $40,40 \pm 2,96$ \\
\hline \multirow{2}{*}{ VCM (fL) } & suplementado & $86,50 \pm 2,90$ & $86,66 \pm 3,15$ & $87,03 \pm 2,84$ \\
\hline & placebo & $86,64 \pm 2,01$ & $85,92 \pm 2,30$ & $86,94 \pm 3,64$ \\
\hline \multirow{2}{*}{ HCM (pg) } & suplementado & $30,11 \pm 1,34$ & $28,64 \pm 1,06^{a}$ & $28,01 \pm 1,23^{a}$ \\
\hline & placebo & $29,35 \pm 0,96$ & $28,36 \pm 0,89$ & $27,32 \pm 1,01^{a}$ \\
\hline \multirow{2}{*}{ CHCM (g/dL) } & suplementado & $34,81 \pm 0,86$ & $33,05 \pm 1,14^{a}$ & $32,18 \pm 0,73^{a}$ \\
\hline & placebo & $33,87 \pm 0,86$ & $32,95 \pm 1,14$ & $31,43 \pm 0,73^{a, b}$ \\
\hline \multirow{2}{*}{ RDW (\%) } & suplementado & $11,66 \pm 0,49$ & $12,27 \pm 0,56$ & $11,95 \pm 0,57$ \\
\hline & placebo & $11,95 \pm 0,49$ & $12,50 \pm 0,56$ & $12,56 \pm 0,57$ \\
\hline
\end{tabular}


T0: basal; T1: primeira semana de treinamento; T2 terceira semana de treinamento. (a) Quando estatisticamente significativo versus T0; $\left({ }^{b}\right)$ Quando estatisticamente significativo versus T1. Os valores estão expressos como média \pm desvio padrão. $P<0,05$.

\section{DISCUSSÃO}

Os indivíduos do presente estudo apresentaram composição corporal compatível com as exigências físicas solicitadas durante a realização do CBP, não sendo observadas diferenças entre os valores médios dos parâmetros analisados com aqueles descritos na literatura como ponto de corte considerado normal (Marfell-Jones et al., 2019; Pollock \& Wilmore, 1993). No início do estudo, todos os militares apresentaram bom estado de saúde e condicionamento físico, exigência para a matrícula no CBP (Brasil, 2013).

Este trabalho investigou o efeito da suplementação antioxidante composta por: Selênio, vitaminas C e E contra a ação das EROs produzidas pelo sobre biomarcadores hematológicos (ex. hemácias, Hb, Hct, VCM, HCM, CHCM e RDW) em militares do CBP submetidos a três semanas de EFIP. Um olhar cuidadoso em nossos resultados nos permitiu inferir que a primeira semana do curso (T1) configura-se como a mais difícil para os alunos, pois foi observado um maior número de desligamentos quando comparada com a terceira semana de treinamento (T2).

0 principal achado deste estudo foi que o regime de suplementação administrado não foi capaz de modificar os efeitos do EFIP sobre os biomarcadores hematológicos. A Hb mostrou-se ser o biomarcador mais suscetível aos efeitos do treinamento, reduzindo significativamente em ambos os grupos durante os períodos do estudo. De fato, estudos apontam que o exercício físico induz um quadro de hemólise com o aumento de $\mathrm{Hb}$ livre no soro (Lippi \& Sanchis-Gomar, 2019; Lücker et al., 2017; Silva et al., 2021). Por outro lado, é sabido que o tempo de vida útil das hemácias de indivíduos fisicamente ativos na circulação sanguínea, principalmente aqueles praticantes de corrida intensa e prolongada, é menor do que em indivíduos sedentários (Mairbäurl, 2013).

Não obstante a este fato, um estudo realizado por Coates et al. (2017) constatou em corredores e triatletas uma maior incidência de anemia por deficiência de ferro acompanhada por uma redução na concentração de Hb. Neste cenário, Tsai et al. (2019) observaram em militares diagnosticados com anemia leve (redução de hemácias, $\mathrm{Hb}$ e Hct), submetidos a uma corrida de 3.000 metros, uma menor aptidão cardiorrespiratória após a corrida. Estes dados reforçam a hipótese da necessidade de mecanismo preventivos, tais como uma suplementação de ferro e/ou antioxidante, a fim de evitar maiores danos na série vermelha do hemograma, evitando assim o comprometimento no aporte de $\mathrm{O}_{2}$ para os tecidos e consequente redução no desempenho físico. 
De fato, Chou et al. (2018) ao suplementarem 18 atletas de taekwondo com as vitaminas C e E, observaram após $24 \mathrm{~h}$ da prática do exercício uma redução na concentração de creatina quinase $(\mathrm{CK})$, resposta inflamatória, mioglobina e hemólise no grupo suplementado quando comparado ao grupo placebo. Neste contexto, alguns estudos evidenciam que o estresse oxidativo desempenha um papel significativo em prover dano a membrana das hemácias, levando a sua deformabilidade (peroxidação lipídica) e, deste modo, recomendam o uso de suplementação antioxidante para atenuar esses danos (Bissinger et al., 2019; Fibach \& Rachmilewitz, 2008; Mohanty et al., 2014). Ao mesmo tempo, é sabido que o exercício intenso induz a redução de nutrientes essenciais ao bom funcionamento do sistema antioxidante, deixando o organismo suscetível ao ataque das EROs (Maynar et al., 2019).

As hemácias e o Hct não apresentaram nenhum tipo de alteração ao longo dos períodos do estudo, talvez pelo fato da amostra ser composta por indivíduos fisicamente ativos, o que pode ter contribuído com uma maior proteção fornecida pelo sistema antioxidante endógeno contra 0 ataque das EROs (Di Meo et al., 2019; Pospieszna et al., 2020). Cabe ressaltar que as atividades de contato com as mãos e a corrida de $5 \mathrm{Km}$ realizada no CBP, não geraram um estresse mecânico suficiente para induzir um quadro de hemólise intravascular. De fato, a literatura descreve que para ocorrer hemólise, durante um período de corrida, os percursos necessitam ser semelhantes àqueles realizados em maratonas e ultramaratonas (Nikolaidis et al., 2018; Sanchis-Gomar et al., 2016). Este resultado também explica por que o VCM não sofreu alteração, pois ele é calculado pela razão entre o Hct e as hemácias, ambos parâmetros não sofreram alterações neste estudo, e permite estimar que o nível de hidratação realizado durante o CBP foi satisfatório.

Ao contrário do VCM, o HCM e CHCM reduziram ao longo do curso. Este resultado foi motivado pela redução na concentração de $\mathrm{Hb}$, pois o HCM é obtido pelo cálculo da razão entre a $\mathrm{Hb}$ e hemácias, e o CHCM pelo cálculo da razão entre a Hb e o Hct. No entanto, ao observarmos a redução de CHCM no GP em T2 versus T1, sugerimos que o GS obteve uma recuperação melhor nos níveis de $\mathrm{Hb}$ do que o GP (Failace, 2015; Hoffbrand \& Steensma, 2020). Após um olhar cuidadoso no percentual de RDW ao longo dos períodos, embora este biomarcador não tenha apresentado diferença estatística, nos levou a sugerir que parece haver uma compensação do organismo com hemácias de tamanho maior no GP, contribuindo para uma variação no volume das hemácias (anisocitose), provavelmente favorecido por um maior aumento na destruição dessas células (Alis et al., 2015; Failace, 2015; Hoffbrand \& Steensma, 2020).

Em linhas gerais, militares estão suscetíveis a danos no metabolismo eritrocitário induzido pelo exercício físico, que podem comprometer a saúde e a qualidade de vida destes indivíduos (Epstein et al., 2018; Myhre et al., 2016; Saidi et al., 2019). Neste sentido, alguns estudos recomendam o monitoramento dos biomarcadores pertencentes a série 
vermelha antes, durante e após períodos de treinamento físico, objetivando prevenir danos ao organismo (Mariño et al., 2020; Pingitore et al., 2015).

0 presente estudo apresenta algumas limitações: i) tamanho do grupo amostral, o que pode ter contribuído por meio da individualidade biológica nas respostas dos biomarcadores; ii) não avaliação da alimentação; iii) a impossibilidade de um período maior de suplementação; iv) falta de análise de alguns biomarcadores importantes na compreensão do processo de hemólise e metabolismo eritrocitário, tais como, eritropoetina, ferro e haptoglobina.

Com base na análise dos resultados encontrados nesse estudo, concluímos que o EFIP induziu uma redução na concentração de $\mathrm{Hb}$ no sangue periférico dos militares voluntários a realização do CBP, porém a suplementação antioxidante administrada composta de selênio e das vitaminas C e E não promoveu efeito contra o dano causado pelo EFIP.

\section{AGRADECIMENTOS}

Os autores agradecem ao Exército Brasileiro pela acessibilidade aos militares voluntários a realização do curso básico paraquedista, e por todo o apoio ao trabalho, bem como a Farmácia de Homeopatia Quintessência Ltda., CGC 311255860003/06, pela confecção das cápsulas. 


\section{BIBLIOGRAFIA}

Aguiló, A., Tauler, P., Sureda, A., Cases, N., Tur, J., \& Pons, A. (2007). Antioxidant diet supplementation enhances aerobic performance in amateur sportsmen. Journal of Sports Sciences, 25(11), 1203-1210. https://doi.org/10.1080/02640410600951597

Ainsworth, B. E., Haskell, W. L., Herrmann, S. D., Meckes, N., Bassett, D. R., Tudor-Locke, C., Greer, J. L., Vezina, J., Whitt-Glover, M. C., \& Leon, A. S. (2011). 2011 compendium of physical activities: A second update of codes and MET values. Medicine and Science in Sports and Exercise, 43(8), 1575-1581. https://doi.org/10.1249/MSS.0b013e31821ece12

Alis, R., Romagnoli, M., Primo-Carrau, C., Pareja-Galeano, H., Blesa, J. R., \& SanchisGomar, F. (2015). Effect of exhaustive running exercise on red blood cell distribution width. Clinical Chemistry and Laboratory Medicine, 53(2), e29-e31. https://doi.org/10.1515/cclm-2014-0749

Balushi, H. Al, Hannemann, A., Rees, D., Brewin, J., \& Gibson, J. S. (2019). The effect of antioxidants on the properties of red blood cells from patients with sickle cell anemia. Frontiers in Physiology, 10(JUL). https://doi.org/10.3389/fphys.2019.00976

Barbosa, K. B. F., Costa, N. M. B., De Cássia Gonçalves Alfenas, R., De Paula, S. O., Minim, V. P. R., \& Bressan, J. (2010). Estresse oxidativo: Conceito, implicações e fatores modulatórios. Revista de Nutricao, 23(4), 629-643. https://doi.org/10.1590/S141552732010000400013

Bissinger, R., Bhuyan, A. A. M., Qadri, S. M., \& Lang, F. (2019). Oxidative stress, eryptosis and anemia: a pivotal mechanistic nexus in systemic diseases. FEBS Journal, 286(5), 826-854. https://doi.org/10.1111/febs.14606

Borg, G. (2000). Escalas de Borg para a dor e o esforço: percebido. São Paulo: Manole.

Brasil. (2013). Ministério da Defesa. Exército Brasileiro. Caderno de Instrução de Treinamento e Técnica Básica do Paaraquedista Militar - EB70-Cl-11.001. 1a ed. file:///C:/Users/mabsm/Downloads/EB70-Cl-11.001 (5).pdf

Brendel, H., Shahid, A., Hofmann, A., Mittag, J., Bornstein, S. R., Morawietz, H., \& Brunssen, C. (2020). Nadph oxidase 4mediates the protective effects of physical activity against obesity-induced vascular dysfunction. Cardiovascular Research, 116(10), 1767-1778. https://doi.org/10.1093/cvr/cvz322

Chou, C. C., Sung, Y. C., Davison, G., Chen, C. Y., \& Liao, Y. H. (2018). Short-term highdose vitamin $\mathrm{C}$ and $\mathrm{E}$ supplementation attenuates muscle damage and inflammatory 
responses to repeated taekwondo competitions: A randomized placebo-controlled trial. International Journal of Medical Sciences, 15(11), 1217-1226. https://doi.org/10.7150/ijms.26340

Coates, A., Mountjoy, M., \& Burr, J. (2017). Incidence of Iron Deficiency and Iron Deficient Anemia in Elite Runners and Triathletes. Clinical Journal of Sport Medicine, 27(5), 493-498. https://doi.org/10.1097/JSM.0000000000000390

Cotinguiba, G. G., Silva, J. ocksane do N., Azevedo, R. R. de S., Rocha, T. J. M., \& Santos, A. F. dos. (2013). Método de Avaliação da Defesa Antioxidante : Uma Revisão de Literatura Methods of the Antioxidant Defense : A Literature Review. UNOPAR Cient., Ciênc. Biol. Saude, 15(3), 231-237.

Cunha, T. F., Bechara, L. R. G., Bacurau, A. V. N., Jannig, P. R., Voltarelli, V. A., Dourado, P. M., Vasconcelos, A. R., Scavone, C., Ferreira, J. C. B., \& Brum, P. C. (2017). Exercise training decreases NADPH oxidase activity and restores skeletal muscle mass in heart failure rats. Journal of Applied Physiology, 122(4), 817-827. https://doi.org/10.1152/japplphysiol.00182.2016

Danese, E., Lippi, G., Sanchis-Gomar, F., Brocco, G., Rizzo, M., Banach, M., \& Montagnana, M. (2017). Physical Exercise and DNA Injury: Good or Evil? In Advances in Clinical Chemistry (1st ed., Vol. 81). Elsevier Inc. https://doi.org/10.1016/bs.acc.2017.01.005

Dasso, N. A. (2019). How is exercise different from physical activity? A concept analysis. Nursing Forum, 54(1), 45-52. https://doi.org/10.1111/nuf.12296

Di Meo, S., Napolitano, G., \& Venditti, P. (2019). Mediators of physical activity protection against ros-linked skeletal muscle damage. International Journal of Molecular Sciences, 20(12), 1-38. https://doi.org/10.3390/ijms20123024

Epstein, D., Borohovitz, A., Merdler, I., Furman, M., Atalli, E., Sorkin, A., Stainfeld, Y., Isenberg, Y., Mashiach, T., Shapira, S., Weisshof, R., \& Dann, E. J. (2018). Prevalence of Iron Deficiency and Iron Deficiency Anemia in Strenuously Training Male Army Recruits. Acta Haematologica, 139(3), 141-147. https://doi.org/10.1159/000485736

Failace, R. (2015). Hemograma: manual de interpretação (Artmed Edi).

Fibach, E., \& Rachmilewitz, E. (2008). Fibach, E., \& Rachmilewitz, E. (2008). The role of oxidative stress in hemolytic anemia. Current molecular medicine, 8(7), 609-619.. 609-619. 
Gammone, M. A., Gemello, E., Riccioni, G., \& D’Orazio, N. (2014). Marine bioactives and potential application in sports. Marine Drugs, 12(5), 2357-2382. https://doi.org/10.3390/md12052357

Gaschler, M. M., \& Stockwell, B. R. (2018). Lipid peroxidation in cell death. Biochem Biophys Res Commun, 176(1), 100-106. https://doi.org/10.1016/j.bbrc.2016.10.086.Lipid

Hasani, M., Djalalinia, S., Khazdooz, M., Asayesh, H., Zarei, M., Gorabi, A. M., Ansari, H., Qorbani, M., \& Heshmat, R. (2019). Effect of selenium supplementation on antioxidant markers: a systematic review and meta-analysis of randomized controlled trials. Hormones, 18(4), 451-462. https://doi.org/10.1007/s42000-01900143-3

Helmut Sies, Carsten Berndt, and D. P. J. (2017). Oxidative Stress. Annual Review of Biochemistry, 86, 715-748. https://doi.org/https://doi.org/10.1146/annurevbiochem- 061516-045037

Hoffbrand, A. V, \& Steensma, D. P. (2020). Hoffbrand's Essential Haematology (8 ed).

Kumar, D., \& Rizvi, S. I. (2014). Markers of oxidative stress in senescent erythrocytes obtained from young and old age rats. Rejuvenation Research, 17(5), 446-452. https://doi.org/10.1089/rej.2014.1573

Lippi, G., \& Sanchis-Gomar, F. (2019). Epidemiological, biological and clinical update on exercise-induced hemolysis. Annals of Translational Medicine, 7(12), 270-270. https://doi.org/10.21037/atm.2019.05.41

Lücker, A., Secomb, T. W., Weber, B., \& Jenny, P. (2017). The relative influence of hematocrit and red blood cell velocity on oxygen transport from capillaries to tissue. Microcirculation, 24(3). https://doi.org/10.1111/micc.12337

Mairbäurl, H. (2013). Red blood cells in sports: Effects of exercise and training on oxygen supply by red blood cells. Frontiers in Physiology, 4 NOV(November), 1-13. https://doi.org/10.3389/fphys.2013.00332

Marengo, B., Nitti, M., Furfaro, A. L., Colla, R., Ciucis, C. De, Marinari, U. M., Pronzato, M. A., Traverso, N., \& Domenicotti, C. (2016). Redox homeostasis and cellular antioxidant systems: Crucial players in cancer growth and therapy. Oxidative Medicine and Cellular Longevity, 2016. https://doi.org/10.1155/2016/6235641

Marfell-Jones, M., Esparza-Ros, F., \& Vaquero-Cristóbal, R. (2019). ISAK accreditation handbook. Murcia: International Society for the Advancement of Kinanthropometry - ISAK. 
Mariño, M. M., Grijota, F. J., Bartolomé, I., Siquier-Coll, J., Román, V. T., \& Muñoz, D. (2020). Influence of physical training on erythrocyte concentrations of iron, phosphorus and magnesium. Journal of the International Society of Sports Nutrition, 17(1), 1-7. https://doi.org/10.1186/s12970-020-0339-y

Maynar, M., Bartolomé, I., Alves, J., Barrientos, G., Grijota, F. J., Robles, M. C., \& Munõz, D. (2019). Influence of a 6-month physical training program on serum and urinary concentrations of trace metals in middle distance elite runners. Journal of the International Society of Sports Nutrition, 16(1), 1-10. https://doi.org/10.1186/s12970-019-0322-7

Mohanty, J. G., Nagababu, E., \& Rifkind, J. M. (2014). Red blood cell oxidative stress impairs oxygen delivery and induces red blood cell aging. Frontiers in Physiology, 5 FEB(February), 1-6. https://doi.org/10.3389/fphys.2014.00084

Müller-Schilling, L., Gundlach, N., Böckelmann, I., \& Sammito, S. (2019). Physical fitness as a risk factor for injuries and excessive stress symptoms during basic military training. International Archives of Occupational and Environmental Health, 92(6), 837-841. https://doi.org/10.1007/s00420-019-01423-6

Myhre, K. E., Webber, B. J., Cropper, T. L., Tchandja, J. N., Ahrendt, D. M., Dillon, C. A., Haas, R. W., Guy, S. L., Pawlak, M. T., \& Federinko, S. P. (2016). Prevalence and Impact of Anemia on Basic Trainees in the US Air Force. Sports Medicine - Open, 2(1), 0-6. https://doi.org/10.1186/s40798-016-0047-y

Nikolaidis, P. T., Veniamakis, E., Rosemann, T., \& Knechtle, B. (2018). Nutrition in ultraendurance: State of the art. Nutrients, 10(12). https://doi.org/10.3390/nu10121995

Ojanen, T., Jalanko, P., \& Kyröläinen, H. (2018). Physical fitness, hormonal, and immunological responses during prolonged military field training. Physiological Reports, 6(17), 1-10. https://doi.org/10.14814/phy2.13850

Padovani, R. M., Amaya-Farfán, J., Colugnati, F. A. B., \& Domene, S. M. Á. (2006). Dietary reference intakes: Application of tables in nutritional studies. Revista de Nutricao, 19(6), 741-760. https://doi.org/10.1590/\$1415-52732006000600010

Pingitore, A., Lima, G. P. P., Mastorci, F., Quinones, A., lervasi, G., \& Vassalle, C. (2015). Exercise and oxidative stress: Potential effects of antioxidant dietary strategies in sports. Nutrition, 31(7-8), 916-922. https://doi.org/10.1016/j.nut.2015.02.005

Pisoschi, A. M., \& Pop, A. (2015). The role of antioxidants in the chemistry of oxidative stress: A review. European Journal of Medicinal Chemistry, 97, 55-74. https://doi.org/10.1016/j.ejmech.2015.04.040 
Poblete-Aro, C., Russell-Guzmán, J., Parra, P., Soto-Muñoz, M., Villegas-González, B., Cofré-Bola-Dos, C., \& Herrera-Valenzuela, T. (2018). Efecto del ejercicio físico sobre marcadores de estrés oxidativo en pacientes con diabetes mellitus tipo 2. Revista Médica de Chile, 146(3), 362-372. https://doi.org/10.4067/s003498872018000300362

Pollock, M., \& Wilmore, J. (1993). Health and disease exercises: assessment and prescription for prevention and rehabilitation (2a ed).

Pospieszna, B., Kusy, K., Słomińska, E. M., Dudzinska, W., Ciekot-Sołtysiak, M., \& Zieliński, J. (2020). The effect of training on erythrocyte energy status and plasma purine metabolites in athletes. Metabolites, 10(1), 1-15. https://doi.org/10.3390/metabo10010005

Rapido, F. (2017). The potential adverse effects of haemolysis. Blood Transfusion, 15(3), 218-221. https://doi.org/10.2450/2017.0311-16

Rifkind, J. M., Mohanty, J. G., \& Nagababu, E. (2015). The pathophysiology of extracellular hemoglobin associated with enhanced oxidative reactions. Frontiers in Physiology, 6(JAN), 1-7. https://doi.org/10.3389/fphys.2014.00500

Saidi, K., Zouhal, H., Rhibi, F., Tijani, J. M., Boullosa, D., Chebbi, A., Hackney, A. C., Granacher, U., Bideau, B., \& Abderrahman, A. Ben. (2019). Effects of a six-week period of congested match play on plasma volume variations, hematological parameters, training workload and physical fitness in elite soccer players. PLoS ONE, 14(7), 1-17. https://doi.org/10.1371/journal.pone.0219692

Sanchis-Gomar, F., Alis, R., Rodríguez-Vicente, G., Lucia, A., Casajús, J. A., \& Garatachea, N. (2016). Blood and urinary abnormalities induced during and after 24-hour continuous running: A case report. Clinical Journal of Sport Medicine, 26(5), e100e102. https://doi.org/10.1097/JSM.0000000000000222

Sies, H. (2018). On the history of oxidative stress: Concept and some aspects of current development. Current Opinion in Toxicology, 7(2018), 122-126. https://doi.org/10.1016/j.cotox.2018.01.002

Silva, A. C. da, Guimarães, T. T., Silva, J. M. da, Gomes, D. V., Mendonça, C. F., Pesquero, J. B., Palmisano, G., Moreira, J. C., \& Pereira, M. D. (2021). Rabdomiólise em militares: uma missão de reconhecimento para prevenção. Military rhabdomyolysis: a reconnaissance mission for prevention. Jornal de Investigação Médica, 2(1).

Sumita, N. M., Andriolo, A., Ferreira, C. E. dos S., Campana, G. A., Oliveira, G., De, F., Brazão, F. V., Vasconcellos, L. de S., Junior, A. P., Lopes, A. C. W., Shcolnik, W., \& Mendes, M. E. (2020). Sociedade Brasileira De Patologia Clínica / Medicina 
Laboratorial (SBPC/ML): Boas Práticas em Laboratório Clínico. In Medicina (Manole Ltd).

Szivak, T. K., Lee, E. C., Saenz, C., Flanagan, S. D., Focht, B. C., Volek, J. S., Maresh, C. M., \& Kraemer, W. J. (2018). Adrenal stress and physical performance during military survival training. Aerospace Medicine and Human Performance, 89(2), 99107. https://doi.org/10.3357/AMHP.4831.2018

Tarafdar, A., \& Pula, G. (2018). The role of NADPH oxidases and oxidative stress in neurodegenerative disorders. International Journal of Molecular Sciences, 19(12). https://doi.org/10.3390/ijms19123824

Tsai, K. Z., Lai, S. W., Hsieh, C. J., Lin, C. S., Lin, Y. P., Tsai, S. C., Chung, P. S., Lin, Y. K., Lin, T. C., Ho, C. L., Han, C. L., Kwon, Y., Hsieh, C. B., \& Lin, G. M. (2019). Association between mild anemia and physical fitness in a military male cohort: The CHIEF study. Scientific Reports, 9(1), 11165. https://doi.org/10.1038/s41598-01947625-3

Valko, M., Jomova, K., Rhodes, C. J., Kuča, K., \& Musílek, K. (2016). Redox- and nonredox-metal-induced formation of free radicals and their role in human disease. In Archives of Toxicology (Vol. 90, Issue 1). https://doi.org/10.1007/s00204-015-15795

Viña, J., Olaso-Gonzalez, G., Arc-Chagnaud, C., De La Rosa, A., \& Gomez-Cabrera, M. C. (2020). Modulating Oxidant Levels to Promote Healthy Aging. Antioxidants and Redox Signaling, 33(8), 570-579. https://doi.org/10.1089/ars.2020.8036

WHO. (2020). guidelines on physical activity and sedentary behaviour. Geneva: World Health Organization; Licence: CC BY-NC-SA 3.0 IGO. https://www.who.int/publications//item/9789240015128. 